\title{
Autorschaft, Auto(r)fiktion und Selbstarchivierung in Clemens J. Setz' Erzählwerk
}

So schwer kann das wirklich nicht sein. (Clemens J. Setz, Bot)

Wer das Erzählwerk des österreichischen Autors Clemens J. Setz überblickt, dem mag auffallen, wie unterschiedliche Bedeutungsaspekte des Archivischen die verschiedenen Konzepte der Autorschaft in seinem Schreiben sukzessive usurpieren. Um diese Beobachtung nachvollziehbar zu machen, soll zunächst in aller Kürze der Versuch unternommen werden, Theoreme aus den entsprechenden Diskursen zusammenzuführen, um in einem zweiten Schritt darauf aufbauend die narratologischen Veränderungen der Schreibhaltung in Setz' Prosawerk, das die Archivfiktion konsequent ins digitale Zeitalter überträgt, herauszuarbeiten.

Autorschaft ist - nach der Zertrümmerung des emphatischen, autonom-schöpferischen Autorbegriffs durch Roland Barthes und Michel Foucault Ende der 1960er Jahre - spätestens seit der Jahrtausendwende wieder ein zentraler Forschungsbereich weit über die Literaturwissenschaft hinaus. ${ }^{1}$ Starke Impulse für den Diskurs ergaben sich zumal durch die Präsentations-, Interaktions- und Aufbewahrungsmöglichkeiten der neuen Medien, die schon früher beobachtbare Phänomene wie „kollektive Autorschaft, Inszenierungsstrategien und Imagebildungen“ (Schaffrick und Willand 2015, 7) intensivieren und in vielfacher Beziehung neue Rahmenbe-

1 Die bereits Anfang der 1990er Jahre v. a. im angloamerikanischen Raum angestoßene und weitergeführte Debatte um Status und Funktion des Autors/der Autorin (vgl. u. a. Burke (1992), Biriotti und Miller (1993), Woodmansee (1994)) wurde gegen Ende des Jahrzehnts mit dem Sammelband von Jannidis et al. (1999) auch in der deutschsprachigen Literaturwissenschaft aufgegriffen. Es folgten seitdem zahlreiche weitere wichtige Beiträge und Sammelbände zur Theorie, historischen Dimensionierung und Rekonzeptualisierung des Autorschaftskonzepts; verwiesen sei in diesem Zusammenhang lediglich auf Detering (2002), Städtke und Kray (2003), Künzel und Schönert (2007), Wagner-Egelhaaf (2012), Bannert und Klecker (2013), Schaffrick und Willand (2015, vgl. dort auch eine Auswahlbibliografie zur Autorschaftsforschung 615-655) sowie Arnold et. al. (2017). 
dingungen für das schreibende Ich schufen. Dass diese neue Vorstellung von Autorschaft im digitalen Zeitalter auch in vielerlei Hinsicht mit dem Archiv sowohl als Institution des sammelnden Bewahrens und Dokumentierens als auch als oszillierende ideengeschichtliche Metapher (vgl. Schenk 2013, 46) zu tun hat, ist nicht zu übersehen. Denn als eine Art Hyperarchiv, das digitale Informationen jedweder Provenienz sammelt, verarbeitet, in Verbindung zueinander bringt und weitgehend frei verfügbar macht, befördert das Internet mit seinen Verarbeitungs-, Distributions- und Rezeptionsstrategien das Entstehen dieser neuartigen Modelle des Autorseins entscheidend.

Zum einen scheint der Aspekt der Urheberschaft unter den Konstituenten der virtuellen Welt immer labiler, diffuser oder kontingenter zu werden. Wer wofür verantwortlich ist, erschließt sich in den identitätsauflösenden Weiten des Internets weniger über traditionelle Kategorien wie Individualität, Originalität, Kreativität oder Authentizität als verstärkt über die dominante Medialität der performativen Inszenierung, vermarktenden Adaption oder schlicht Aneignung (wie etwa der Fall Helene Hegemann eindrücklich zeigte). Parallel dazu differenzieren sich - zweitens - die Möglichkeiten der Inszenierung von Autorschaft durch die erleichterte Zugänglichkeit des literarischen Felds, die schwindende Bedeutung von Gatekeepern in Produktion, Vertrieb und Kritik oder auch durch die paratextuelle Vielstimmigkeit im Netz immer weiter aus. Wer als Autor/in wahrgenommen werden will, hat dazu wesentlich mehr Möglichkeiten als je zuvor; mangelnde Qualität oder fehlende Förderung sind in Zeiten von Blogs, SelfPublishing-Plattformen oder Books-on-Demand keine Ausschlusskriterien mehr. Dazu kommt - drittens -, dass immer mehr Schreibende das Hyperarchiv Internet als Medium der Selbst- und Welterfahrung, der Recherche, Weiterbildung, Sinnstiftung und Multiplikation in den Mittelpunkt ihrer Arbeit stellen und so ein immer komplexeres intermediales Referenzsystem etablieren mit den entsprechenden Auswirkungen auf die Identifikation von Autorsubjekt bzw. Autorsubjektkonstruktionen und Erzählinstanz.

Eine Literatur, die diese veränderten Konstellationen produktiv verarbeitet, trägt im Sinne der Kulturökonomik von Boris Groys essenziell zu einem kulturellen Archiv bei, in dem das alltägliche Neue in Interaktion mit dem Etablierten aufgenommen und aufgewertet wird. Moritz Baßler hat vor einiger Zeit diese literarische Archivierungsleistung, die das Profane, Banale und Abseitige mit den damit verbundenen Assoziationen im kulturell Valorisierten verankert, als Kennzeichen des Pop-Romans definiert (vgl. Baßler 2005, 135-136). Man könnte diese Literarisierung des Alltäglichen, die geläufige Werthierarchien unterwandert, jedoch nicht allein auf dieses Genre bzw. dieses Etikett beschränken, sondern es als symptomatisch für ein - ich nenne es - ,archivarisches‘ Autorschaftskonzept 
werten, dem sich unter den Bedingungen der digitalen Welt neue Perspektiven und Ausdrucksformen eröffneten.

Eine besondere Brisanz gewinnt dieses Konzept bei autofiktionalem Schreiben, also bei Texten, in denen Autobiografisches mit Fiktionalem zu einem Identitätsspiel verwoben wird, das die Grenzen zwischen Autorschaft und Erzählinstanz verschwimmen lässt (vgl. Wagner-Egelhaaf 2012; Krumrey 2015). Als selbstreferenzielles Zentrum des Textes literarisiert bzw. fingiert der konkrete Autor Ereignisse und Fakten seines Umfelds, deren Realitätsnähe und Unmittelbarkeit Glaubwürdigkeit suggerieren, doch unklar lassen, inwieweit wir es tatsächlich mit autobiografischem, quasi-faktualem Erzählen oder doch mit Erfundenem zu tun haben. Worin das ,Autorkonstrukt‘ als Resultat eines semiotischen Leseakts mit dem konkreten Autor als realhistorischer Persönlichkeit übereinstimmt, lässt sich nicht zweifelsfrei benennen. Der Versuch, das - vorgeblich eigene Erleben und Denken in Form erzählender Texte $\mathrm{zu}$ archivieren, unterminiert somit auch die in germanistischen Seminaren so essenzielle und dementsprechend vehement eingeforderte Unterscheidung zwischen Autor/in, Erzählinstanz und Figur, weil sie die klare Trennung von deskriptivem, hermeneutischem und einordnend-wertendem Zugang, von Textanalyse, Textinterpretation und Literaturkritik erschwert, wenn nicht verunmöglicht.

\section{2}

Wie sich das narratologisch zu Buche schlägt, lässt sich besonders instruktiv am Erzählwerk des 1982 in Graz geborenen Autors Clemens J. Setz zeigen, der seit mehr als einem Jahrzehnt zu den Lieblingen der deutschsprachigen Literaturkritik zählt, von der Literaturwissenschaft allerdings erst allmählich als dankbares Untersuchungsobjekt entdeckt wird. ${ }^{2}$ Die Verschränkung von Fund, Erfundenem und Befund, die die auktoriale Empirie zum Angelpunkt eines Kosmos der Bizarrerien werden lässt, gehört seit seinem Debüt zu den grundlegenden Schreibverfahren des entsprungenen Germanistikstudenten. Essenzieller Teil seiner Selbstinszenierung ist die zunehmende Verwischung von Autor- und Erzählinstanz, durch die das schreibende Ich stets gegenwärtig zu sein scheint, ohne sich dadurch greifbar, angreifbar zu machen. Wie sehr das Spiel mit der eigenen Identität Strategie seines Schreibens ist, lässt Setz des Öfteren in Interviews und poetologischen Reflexionen anklingen. Im Vorwort seines Pseudo-Interview-

2 Für einen Überblick zur literaturkritischen und -wissenschaftlichen Sekundärliteratur bis April 2020 s. Reichmann 2020. 
bands Bot konstatiert er etwa in Anspielung an Douglas Hofstadters I Am a Strange Loop, „dass man auch mich dereinst würde rekonstruieren können aus dem Material, das ich hinterlassen habe. So schwer kann das wirklich nicht sein“ (Setz 2018, 9). Die Selbstdarstellungsweisen der Autofiktion könnten also nach Setz als Basis einer zukünftigen Autorfiktion dienen, die den angeblich wahren Autor aus der Fiktionalität seines schreibenden Ich nachbildet. Zugegeben eine mutige Vorstellung für einen Literaten, der - glaubt man der Literaturkritik - in seinem Werk vor allem mit ,Shock and Awe` zu punkten wisse, mit einer ,Ästhetik der Drastik‘, die die Grenzen des guten Geschmacks hinsichtlich Sexualität, Gewalt und Ekel geflissentlich ignoriert (vgl. Oberreither 2020, 125-126).

Den Ausgangspunkt dieser literarischen Selbstarchivierung und in weiterer Folge Selbstmodellierung setzte Setz mit seinem Romanerstling Söhne und Planeten, dessen vier ineinander verwobene Erzählungen sich an verschiedenen VaterSohn-Beziehungen abarbeiten. Man könnte meinen: Keine allzu innovative Motivwahl für einen Jungautor, zumal auch die aufdringliche Kafka-Referenz zu Beginn, Unzulänglichkeitsgefühle in Körpertransformationen auszudrücken, etwas abgedroschen daherkommt. Der Schriftsteller René Templ neigt in der Eingangserzählung zu rätselhaften Schrumpfanfällen, die er mit der Lektüre von Daniel Defoes Tagebuch des Pestjahrs zu bekämpfen sucht, ein Buch von 1722, das einen auf Recherchen und Aufzeichnungen beruhenden Augenzeugenbericht des Seuchenjahrs 1665 gibt, der aufgrund seiner Eindringlichkeit lange für authentisch gehalten wurde. Das Changieren zwischen Authentizität und Erfundenem ist denn auch der eigentliche Reiz des Buchs, das aus wechselnden Erzählperspektiven und in verschiedenen, geschickt geschachtelten Konfliktkonstellationen Facetten des Literaturbetriebs reflektiert. In welchen der vielen schreibenden jungen und alten Protagonisten sich das Autor-Ich am deutlichsten verewigt, bleibt freilich unklar: Ist es der jüngere Templ der zweiten Erzählung, der nun autodiegetisch seine Schreibsozialisation mit Anmerkungen wie der folgenden beschreibt?

Wie alle Schriftsteller, die in der Gesellschaft von Frauen aufgezogen worden sind und die ihre Sexualität aus Lust und Notwendigkeit in ihr Werk integrieren, habe ich eine Art von selbstverständlicher Obsession für weibliche Verhaltensweisen, Körper, Beziehungen und Eigenschaften. (Setz 2007, 103)

Oder ist es der Schriftstellersohn Victor Senegger, der Ich-Erzähler der Schlusssequenz, der demnächst ,eher längere Novellen, nicht mehr als drei oder vier, die miteinander zusammenhängen“ (Setz 2007, 203), veröffentlichen will und dessen erratische Texte der lieblose Vater nach dem Selbstmord des Sohns - als kläglicher Versuch der Wiedergutmachung - herausgeben will? Oder sind es seine 
Freunde, die wie „so viele andere junge Menschen in dieser harmlosen und phantasiehungrigen Stadt Schriftsteller“ (Setz 2007, 187) sind? Wichtiger als autofiktionale Vergegenwärtigung ist in diesem Debütroman noch die selbstreflexive Auseinandersetzung mit dem Schreib- und Veröffentlichungsprozess selbst, die Dekuvrierung des intertextuellen Anspielungsreichtums, der Umgang mit Leitbildern wie „Kafka oder ... Haushofer“ (Setz 2007, 199), die Berechenbarkeit der Literaturkritik und ähnliches mehr.

Wesentlich ausgeprägter sind selbstreferenzielles Schreiben und Autofiktion bereits in Setz' zweitem Roman Frequenzen, den er 2009 wieder im ResidenzVerlag vorlegte, und nicht erst bei den Schlussszenen des 700-Seiten-Wälzers, wo uns „Ein junger, ernster Mann mit Brille“ begegnet, der über „das uralte Elementarproblem von Vater und Sohn [...] so lange nachgedacht hatte, bis er schließlich einen quirlig-verzweifelten Roman darüber geschrieben hatte“ (Setz 2009, 695). Schon außerhalb des Buchblocks informiert uns ein auf den Vorsatzblättern quer gedruckter selbstironischer Paratext über den Autor und sein vorliegendes Werk. In einem Eintrag aus dem - selbstredend fingierten - Konversationslexikon der Jenseitsmythen wird „Setz, Clemens Johann“ als „Verfasser obskurer Novellen und Romane“ (Setz 2009, [715 und 716]) charakterisiert, eine onomastische Parallele zu Kafkas Werk gezogen und nach einer Kurzcharakterisierung der Protagonisten eine erste Rezension des Romans als augenzwinkerndes Mise en abyme mitgeliefert. Bemerkenswert ist dabei in unserem Zusammenhang der letzte Absatz auf dem hinteren Einbandspiegel:

An mehreren Stellen des Romans hat sich der Autor $S$. selbst verewigt. Was er damit ausdrücken wollte, ist nicht überliefert. Vielleicht nur, dass man ihn, wenn er nicht existieren würde, kurzerhand er- (Setz 2009)

Hier bricht der Artikel am Seitenende ab. Sollte bereits diese Leerstelle ein Hinweis auf die Erschließbarkeit des Autor-Ich aus seinem Werk sein, der sogenannte implizite Autor also den realen Autor ,er-setz-en‘? Tatsache ist, dass auch in diesem für den Deutschen Buchpreis shortgelisteten Roman die zentralen, wieder ödipal belasteten Protagonisten Alexander und Walter Rückschlüsse auf den Urheber provozieren, da sie - wie immer in Setz' Texten - ähnliche Fähigkeiten, Faibles oder Fehlleistungen haben, wie man sie durch suggestive Fokalisierung und geschickt gestreute Eigenaussagen vom Autor Clemens Setz zu kennen glaubt. So ist der fantasiebegabte Ich-Erzähler Alexander Kehrfuchs, ein verlassener Lehrersohn und Altenpfleger auf Sinnsuche, Ton-Farb-Synästhetiker, wie das auch Setz von sich behauptet. Seine akustische Wahrnehmung beeinflusst also visuelle Eindrücke, was zu hoch- (oder in den Augen gestrenger Kunstrichter 
auch pseudo-)poetischen Formulierungen (vgl. Reichmann 2017, 148) führen kann wie ein Beispiel vom Anfang des Romans nachdrücklich belegt:

Der Dienstag ist ein alter Mann mit Blumen am Hut, sehr gelb im Gesicht, und seine Augen sind fast nur ein Zwinkern. Das Gelb erinnert an die Farbe von giftigem Weizen, eine albtraumhafte Schattierung von dunklem Gold. Der Mittwoch hat die seltsamste Farbe, wahrscheinlich, weil er als einziger Tag der Woche nicht auf die helle Silbe -tag endet. Er ist gesprenkelt ein wenig wie ein Wollknäuel aus verschiedenfarbigen Fäden. (Setz 2009, 18-19)

Synästhesie ist nur eines der vielen neurologischen oder psychologischen Phänomene, mit denen sich Setz inner- und außerhalb seines Schreibens auseinandersetzt (vgl. Oberreither 2019, 128). Sie geben ihm u. a. Gelegenheit, die subtilen, sprachgewaltig umgesetzten Alltagsbeobachtungen mit surreal-witzigen Parallelwelten zu kombinieren. So benötigen Setz' Romane bei aller Umfänglichkeit auch keine Vielfalt an Schauplätzen oder Personen. Seine Kunst liegt in der vielschichtigen Vernetzung unterschiedlichster Informationen zu einem komplexen Ganzen, das weitere Anknüpfungspunkte liefert, nach innen, aber auch nach außen. Wie stark dabei die Rolle des Autors als ästhetischer Katalysator seiner lebensweltlichen Erfahrung dient, macht der beigepackte,Warnhinweis‘ am Ende des Romans deutlich:

Alle realen Personen, die sich in den Figuren dieses Romans wiederfinden, werden durch den Akt der Identifikation zwangsläufig fiktiv und zu einem reinen Produkt meiner Fantasie. (Setz 2009, 714)

Selbst zum Produkt seiner Fantasie macht sich Setz in seiner Erzählung Das Herzstück der Sammlung aus dem mit dem Preis der Leipziger Buchmesse prämierten Prosaband Die Liebe zur Zeit des Mahlstädter Kindes, den er 2011 in seinem neuen Verlag Suhrkamp folgen ließ. Selbstreferenziell ist nicht nur der Titel dieses Texts, der inmitten zumeist verstörend abgründiger Geschichten über Grausamkeit, Krankheit und Perversion deprivierter und depravierter Figuren steht. Auch der Autor selbst inszeniert sich als Herzstück einer Sammlung, die über und um ihn angelegt wurde, allerdings als altes Alter Ego. Wir sind Zeugen der letzten Führung in einem zukünftigen Clemens-Setz-Archiv, das demnächst einer privaten Sammlung einverleibt werden soll, wie der Archivar erläutert. Das Spätwerk, „ein langes Regal voll dunkler, teilweise zerfallener Bücher“, hat er als letztes Highlight seiner Führung der jungen Besucherin bereits präsentiert, den ganzen ,späten Setz', den „Warteschlangen-Zyklus. Enkel und Asteroiden. Alles aus seiner NachMeer-Periode. Aus der Vor-Meer-Periode haben wir nur die Romane, die meisten anderen Texte von damals sind verloren" (Setz 2011, 196). Dereinst verloren müsste damit wohl auch die vorliegende Geschichte sein, in der sich der Setz-Fan 
nun Zutritt zum Metaobjekt der Sammlung verschafft: Verborgen in einem miefigen Nebenraum vegetiert der nur mehr zu Grunzlauten fähige Autor Setz als ,verwitterter' Greis in einem gelben Gitterbett vor sich hin, ein kitschiger Zimmerbrunnen simuliert das für sein Werk angeblich so wesentliche Meer. Die autofiktionale Selbstarchivierung - hier in einer personifizierten SciencefictionVariante - setzt die autobiografischen Elemente nur mehr als Anspielungshorizont eines wenig verheißungsvollen zukünftigen Dichterkults, bei dem Autor und Werk nicht mehr zu sagen haben als ein langgezogenes „Maah“ (Setz 2011, 206).

Gleichsam kompensatorisch zum verlorenen Weltbezug versucht Setz' folgender Roman Indigo (2012) umso nachdrücklicher Authentizität zu suggerieren: durch einen autobiografistischen Ich-Erzähler, über Faktizität imitierende metafiktionale Texte oder auch über die fingierte Materialität angeblich existierender Dokumente, um mit diesen - altbekannten - narratologischen Kunstgriffen die Verschleifung von Autor- und Erzählinstanz zu intensivieren. Freilich ist es keine Wohlfühlwelt, in die sich der Autor Setz als Erzähler und Protagonist versetzt, sondern eine mit sozialvoyeuristischer Lust am Leid entfaltete Dystopie der verstörenden Dispositionen, bedrohlichen Psychosen und allgegenwärtigen Krankheiten. Schon im Vorfeld der Veröffentlichung begann der Autor seine autofiktionale Inszenierung in einem vom Verlag eingerichteten Blog (www.indigo. suhrkamp.de), der den Entstehungsprozess begleitete, um dann das Spiel mit der (Unzu-)Verlässlichkeit des Erzählens in den peritextuellen Interferenzen von Klappentext und Autorenkurzporträt weiterzuspinnen:

Im Norden der Steiermark liegt die Helianau, eine Internatsschule für Kinder, die an einer rätselhaften Störung leiden, dem Indigo-Syndrom. Jedem, der ihnen zu nahe kommt, befallen Übelkeit, Schwindel und heftige Kopfschmerzen. Der junge Mathematiklehrer Clemens Setz unterrichtet an dieser Schule und wird auf seltsame Vorgänge aufmerksam: Immer wieder werden Kinder in eigenartigen Maskierungen in einem Auto mit unbekanntem Ziel davongefahren. Setz beginnt, Nachforschungen anzustellen, doch er kommt nicht weit; er wird aus dem Schuldienst entlassen. Fünfzehn Jahre später berichten die Zeitungen von einem aufsehenerregenden Strafprozess: Ein ehemaliger Mathematiklehrer wird vom Vorwurf freigesprochen, einen Tierquäler brutal ermordet zu haben.

So könnte die Zusammenfassung einer Handlung lauten, die sich jeder Zusammenfassung entzieht. Am besten, Sie blättern gleich um und lesen das Buch. Sie werden feststellen: Auch im neuen Roman von Clemens J. Setz geht das „radikale Gegenprogramm zur hübsch verkasteten Literaturwerkstättenliteratur“ (Die Welt) weiter. Rasend spannend und so erholsam wie eine gute Massage. Hinterher spüren sie jeden Muskel.

Clemens J. Setz wurde 1982 in Graz geboren. Nach dem Studium der Mathematik und Germanistik in Graz arbeitete er als Mathematik-Tutor u. a. im Proximity Awareness \& Learning Center Helianau und als Journalist. Seit 2008 treten bei ihm Spätfolgen der Indigo-Belastung auf. Heute lebt er als freier Schriftsteller zurückgezogen mit seiner Frau in der Nähe von Graz. (Setz 2012) 
Als autotherapeutische „Ablenkungsgeschichte“ (Setz 2012, 293) der homodiegetischen Erzählinstanz Setz wird in Heterodiegese die in die Zukunft (2021) verlegte Geschichte des ehemaligen ,Indigo-Kinds‘ Robert Tätzel vermittelt, der sich und seine Mitmenschen als Außenseiter durch den Alltag quält und schließlich auf seinen ehemaligen, des Mordes bezichtigten Lehrer Setz trifft. Die beiden mit einer Vielzahl an Textsorten angereicherten Erzählstränge generieren eine komplexe (auch typografisch vielseitige) Komposition, die nicht nur „die Grenzen zwischen Facts und Fakes in einer Art literarischem ,Uncanny Valley““ (Reichmann 2017, 152) verschwimmen lässt, sondern eine „regelrecht aberwitzige autofiktionale Dopplung“ kreiert: „Der reale Setz schreibt mit Indigo einen Roman mit einer Hauptfigur Setz, die wiederum eine Geschichte schreibt, in der die Figur Setz auftaucht: eine Autofiktion in einer Autofiktion“ (Pottbeckers 2017, 192). Es ist kein schmeichelhaftes Selbstbild, das hier aus vielfachen Blickwinkeln erstellt wird, aber ein derart kurioses, ja zuweilen beinah karikatureskes, dass man sich stets versucht fühlt, die geschickt ausgelegten Authentifizierungssignale auf ihre Gültigkeit zu überprüfen, um Übereinstimmungen bzw. Abweichungen von Autor, Ich-Erzähler und Setz-Figur auszuloten. Doch zu listig sind die Uneindeutigkeiten unter das Verifizier- und Falsifizierbare gemischt, als dass sich hier Klarheit schaffen ließe - zumal auch die zahlreichen archivierten Inhalte der verlagsinternen Indigo-Internetseite die autofiktionale Inszenierung weiterspinnen.

Noch weitaus stärker warf Suhrkamp die mediale Maschinerie zur Bewerbung des nächsten Romans Die Stunde zwischen Frau und Gitarre (2015) an. Über den ,Blog für Betreutes Lesen' auf der kurzlebigen Social-Reading-Plattform ,Sobooks` sollte der erwartungsgemäß mit Bizarrerien überbordende 1000-Seiten-Klotz von Online-MultiplikatorInnen besprochen, kommentiert und der ebenso anspielungsreiche wie auf Abwege führende Setz'sche Erzählkosmos dechiffriert werden (vgl. Reichmann 2017, 159). Der letztlich überschaubare Zuspruch mag auch dem partiellen Rückzug des Autors geschuldet sein, der sich in diesem Werk weniger outriert, sondern seine Gedankenwelt über die Reflektorfigur der Behindertenpädagogin Natalie Reinegger entfaltet, deren Klient Dorm von seinem ehemaligen Stalkingopfer Hollberg subtil gequält wird. Dass die hypersensible Neurotikerin und Synästhetikerin mit ihrem Faible für sinnverweigernde Nonseq-Gespräche, bulimischen Medienkonsum oder das Abdriften in virtuelle Ablenkungen wie ASMR-Videos oder Computerspiele mit zu entdeckenden Codefehlern unmissverständlich Neigungen und Faszinationen des Autors teilt, hatte dieser mit Essays zur Poesie des ASMR bzw. zur Poesie der Glitches schon vorab klargestellt. Doch vermag die heterodiegetische Vermittlung weniger die Begehrlichkeit des Lesenden zu wecken, ,Autorsuggestionen' als semantische Schnittmengen von Autordisposition und Figurencharakterisierung zu dekuvrieren, selbst wenn Natalie so Setz - durchaus als symbiotische Projektion verstanden werden kann (vgl. 
Leinen 2015). Soziopathien, Borderline-Symptome oder Perversionen wie die Blowjob- und Ejakulat-Manie der Protagonistin, die in der Autofiktion noch als selbstironisches Spiel mit Identitäten ausgelegt werden könnten, ließen sich hier auch als sexistische, gynaikophobe Auswüchse eines Erzählers auslegen, „der so unzweifelhaft ein Mann ist“ (vgl. Kastberger 2016, 22). Da hilft nur bedingt, dass sich Setz in diesem erzähltechnisch vergleichsweise traditionellen Roman mit Natalies Ex-Freund Markus C. Haase zumindest einen schrullig-unbeholfenen schriftstellernden Stellvertreter geschaffen hat, der als verletzlicher Chatpartner, poetologischer Stichwortgeber und leidenschaftlicher John Updike-Verehrer für seine Verhältnisse beinah irritierend normal wirkt. Updikes Tod hatte Markus zutiefst erschüttert, weil damit auch dessen literarische Selbstarchivierung ihr endgültiges Ende fand:

Für Markus waren seine Lieblingsautoren eine spezielle Untergattung der Menschheit, die sich so weit in das Mysterium des Lebens hineingewagt hatten, dass ihr Tod einen persönlich angriff und schmerzte, als verlöre man einen alten Freund. Diese Leute waren ganz allein mit ihren Gedanken über das Sonnensystem und die merkwürdige Einrichtung der Lebensphasen und mit ihrer Angst vor dem Tod spätnachts, aber man wusste das über sie, weil sie es aufschrieben und pausenlos weitererzählten, während man über die meisten anderen Menschen nur Vermutungen anstellen konnte. (Setz 2015, 454)

Auch wenn man Literatur - wie hier - als Festschreiben (und damit Archivierung) der jeweiligen Gedankenwelt versteht, die im cartesianischen Sinn ja Bedingung der eigenen Existenz ist, weist das aus der Lektüre gewonnene Autorkonstrukt stets eine prononcierte Fiktionalität auf, die den Abgleich mit der realen Autorbiografie durch Publikum, Kritik und Wissenschaft geradezu herausfordert.

Dass Setz allerdings kein großes Interesse daran hat, seine kunstvoll konstruierten Identitätssetzungen zu vereindeutigen, ist Angelpunkt seiner bereits angesprochenen Textsammlung Bot, ein Gespräch ohne Autor - wie es im Untertitel heißt -, das Angelika Klammer 2018 herausgab. Der bewerbende Klappentext umreißt die Ausgangskonstellation:

Nachrichten von der ausgelagerten Seele

Hier lesen Sie das Interview mit einem bekannten Schriftsteller - allerdings ohne den Schriftsteller selbst. Denn der war nicht imstande, auf die Fragen der Interviewerin zu antworten. Ihm fiel nichts ein. Zum Glück fand sich eine Alternative: der Clemens-Setz-Bot, eine Art künstliche Intelligenz, sein Millionen von Zeichen umfassendes Tagebuch. Es gibt in verblüffender Offenheit preis, was Sie schon immer über den Autor Clemens J. Setz und sein Werk wissen wollten. (Setz 2018) 
Die vollmundige Ankündigung des Verlags hält freilich nur bedingt, was sie verspricht. Die Suggestion, dass ein Computerprogramm die interpersonelle Kommunikation oder die Repliken des Interviewten ersetzt und der Autor somit nur noch als intellektuelles Materiallager einer ausgelagerten Ordnungsmacht fungiert, ist ebenso irreführend wie das Versprechen, Klarheit über den Autor zu schaffen. Die Fragen orientieren sich vielmehr an den Gedankensplittern des Journals und werden von den handverlesenen oder auch per Suchbegriff zugeordneten Textstellen allenfalls assoziativ, zumeist aber gar nicht beantwortet. Natürlich ist der Urheber dem Gespräch nicht abhandengekommen, wie der Untertitel glauben machen will. Er ist mit seinen oft sehr persönlichen Notizen in der Interaktion mit dem Publikum vielleicht sogar präsenter als in den größeren Kompositionen und fügt dem schillernden Autorkonstrukt Facetten hinzu, die es weiter irrlichtern lassen.

Die im Vorwort zu Bot formulierte Vorstellung, dass sich eine Persönlichkeit aus der Gesamtheit des archivierten Materials rekonstruieren ließe, hebt nicht auf eine herkömmliche Urheber-Biografie ab, sondern versucht ambigen Lebensentwürfen in einer als kontingent erfahrenen digitalisierten Welt gerecht zu werden. Doch wie viel Setz steckt tatsächlich in den Entsetzlichkeiten seines Erzählkosmos? Auch in seinem Kurzgeschichtenband Der Trost runder Dinge (2019) erschließen exzentrische Surrogate der Dichteridentität wieder Bereiche des Ab- und Paranormalen, deren Verquertheit uns erschaudern lassen mag, zugleich aber eine beruhigende Normalität der eigenen Lebenswelt suggeriert. Muss man sich deswegen Sorgen machen um den Autor, dass das autofiktionale Konstrukt der literarischen Selbstarchivierung mit seinem eigentlichen Ich besser übereinstimmt als zu wünschen wäre? Immerhin räsoniert Setz in seiner ,Klagenfurter Rede zur Literatur 2019`Kayfabe und Literatur über nach Drehbuch inszenierte fiktionale Charaktere und Storylines der Wrestlingwelt, die Wrestler zuweilen so internalisieren, dass sie zur eigentlichen Identität werden, und ergänzt:

Die lächerliche Kayfabe der Dichteridentität habe ich selbst besonders hündisch befolgt in meinen jungen Jahren. Vielleicht neigt meine Seele ja allgemein dazu, immer wieder in derlei bereitstehende Hohlformen zu fallen, um sich dann, Jahre später, mühevoll daraus freiknabbern zu müssen. (Setz 2019, 27)

Man sieht: So einfach, wie Setz schelmisch insinuiert, wird seine Rekonstruktion aus dem vorliegenden Material ganz sicher nicht werden. Es scheint paradox, doch hat sich der Autor gerade durch die exzessive selbstreferenzielle Exponierung von autobiografischem und autofiktionalem Material inzwischen eine 
gewisse Aura der Unnahbarkeit erarbeitet. Seine vielgerühmte Schreibweise, die sich die sukzessive Realitätsauflösung einer virtuell überformten digitalen Welt zunutze macht, lässt ein literarisches Setz-Archiv entstehen, das sich aus den unterschiedlichsten Quellen speist und so ein kaleidoskopisch ambivalentes, aus irrwitzigen Details zusammengesetztes Autorbild schafft, das sich zunehmend der Konkretisierung verweigert. Dieser mit jeder Seite erweiterte Setzkasten zusammengetragener und ausgestellter Objets trouvés aus dem eigenen Alltag und/oder den Weiten des Internets ist somit nicht bloß Ergebnis eines zeitgemäßen (selbst)archivarischen Autorschaftskonzepts, sondern wirkt auch erfolgreich als Immunisierungsstrategie gegen die Zumutungen eines gängigen Autorkults.

\section{Literaturverzeichnis}

Arnold, Sonja, Stephanie Catani, Anita Gröger, Christoph Jürgensen, Klaus Schenk und Martina Wagner-Egelhaaf (Hg.). Sich selbst erzählen. Autobiographie - Autofiktion - Autorschaft. Kiel: Ludwig, 2018. 361-377.

Bannert, Herbert, und Elisabeth Klecker (Hg.). Autorschaft. Konzeptionen - Transformationen Diskussionen. Wien: Präsens, 2013.

Detering, Heinrich (Hg.). Autorschaft. Positionen und Revisionen. Stuttgart: Metzler, 2002.

Dinger, Christian. „Die Ausweitung der Fiktion. Autofiktionales Erzählen und (digitale) Paratexte bei Clemens J. Setz und Aléa Torik“. Sich selbst erzählen. Autobiographie - Autofiktion Autorschaft. Hg. Sonja Arnold, Stephanie Catani, Anita Gröger, Christoph Jürgensen, Klaus Schenk und Martina Wagner-Egelhaaf. Kiel: Ludwig, 2018. 361-377.

Baßler, Moritz. Der deutsche Pop-Roman. Die neuen Archivisten. 2. Aufl. München: C. H. Beck, 2005.

Biriotti, Maurice, und Nicola Miller (Hg.). What is an Author? Manchester: University Press, 1993.

Burke, Séan. The Death and Return of the Author. Criticism and Subjectivity in Barthes, Foucault and Derrida. Edinburgh: University Press, 1992.

Kupczyńska, Kalina. „,Einfluss‘ und seine Frequenzen in der Postmoderne. Zur Prosa von Clemens J. Setz“. Zwischen Einflussangst und Einflusslust. Zur Auseinandersetzung mit der Tradition in der österreichischen Gegenwartsliteratur. Hg. Joanna Drynda, Alicja Krauze-Olejniczak und Stawomir Piontek. Wien: Praesens, 2017. 23-33.

Jaklová, Helena. „Im Prosalabor von Clemens J. Setz“. Experimentierräume in der österreichischen Literatur. Hg. Alexandra Millner, Dana Pfeiferová und Vincenza Scuderi. Pilsen: Westböhmische Universität Pilsen, 2019. 308-328.

Jannidis, Fotis, Gerhard Lauer, Matías Martínez und Simone Winko (Hg.). Die Rückkehr des Autors. Tübingen: Niemeyer, 1999.

Jaszi, Peter, und Martha Woodmansee (Hg). The Construction of Authorship. Textual Appropriation in Law and Literature. Durham: Duke University Press, 1994.

Kastberger, Klaus. „Being Clemens Setz. Laudatio“. Clemens J. Setz trifft Wilhelm Raabe. Der Wilhelm Raabe-Literaturpreis 2015. Hg. Hubert Winkels. Göttingen: Wallstein 2016, 14-24. 
Krumrey, Birgitta. Der Autor in seinem Text. Autofiktion in der deutschsprachigen Gegenwartsliteratur als (post-)postmodernes Phänomen. Göttingen: V\&R unipress, 2015.

Künzel, Christine, und Jörg Schönert (Hg.). Autorinszenierungen: Autorschaft und literarisches Werk im Kontext der Medien. Würzburg: Königshausen \& Neumann, 2007.

Leinen, Angela. „Lost in Natalie. Neuer Roman von Clemens J. Setz“. taz.de vom 6. September 2015, https://taz.de/Neuer-Roman-von-Clemens-J-Setz/!5228449/ (15. Juli 2020).

Oberreither, Bernhard. „Irritation - Struktur - Poesie. Zur Poesie erzählter Welten bei Clemens Setz“. Dossier Graz 2000+. Neues aus der Hauptstadt der Literatur. Hg. Gerhard Fuchs, Stefan Maurer und Christian Neuhuber. Graz 2019.125-143, https://unipub.uni-graz.at/ dossier/periodical/titleinfo/4672618 (15. Juli 2020).

Pottbeckers, Jörg. Der Autor als Held. Autofiktionale Inszenierungsstrategien in der deutschsprachigen Gegenwartsliteratur. Würzburg: Königshausen \& Neumann, 2017.

Reichmann, Wolfgang. „Clemens J. Setz“. Junge und jüngere Literatur aus Österreich. Hg. Kalina Kupczyńska. München: edition text + kritik, 2017. 143-164.

Reichmann, Wolfgang. „Clemens J. Setz“. nachschlage.NET/KLG - Kritisches Lexikon zur deutschsprachigen Gegenwartsliteratur, http://www.nachschlage.net/ search $/$ document?index $=$ mol-16\&id $=16000000755$ \&type=text $/ \mathrm{html} \&$ query. key=ut1YDmr9\&template=/publikationen/klg/document.jsp\&preview= (15. Juli 2020).

Schaffrick, Matthias, und Marcus Willand (Hg.). Theorien und Praktiken der Autorschaft. Berlin, Boston: de Gruyter, 2015.

Setz, Clemens J. Söhne und Planeten. St. Pölten, Salzburg: Residenz, 2007.

Setz, Clemens J. Die Frequenzen. St. Pölten, Salzburg: Residenz, 2009.

Setz, Clemens J. Die Liebe zur Zeit des Mahlstädter Kindes. Berlin: Suhrkamp, 2011.

Setz, Clemens J. Indigo. Berlin: Suhrkamp, 2012.

Setz, Clemens J. Die Stunde zwischen Frau und Gitarre. Berlin: Suhrkamp, 2015.

Setz, Clemens J. Bot. Gespräch ohne Autor. Hg. Angelika Klammer. Berlin: Suhrkamp, 2018.

Setz, Clemens J. Der Trost runder Dinge. Berlin: Suhrkamp, 2019.

Setz, Clemens J. Kayfabe und Literatur. Klagenfurter Rede zur Literatur 2019. Klagenfurt: Heyn, 2019.

Städtke, Klaus, Ralph Kray und Ingo Berensmeyer (Hg.). Spielräume des auktorialen Diskurses. Berlin: Akademie Verl., 2003.

Wagner-Egelhaaf, Martina (Hg.). Auto(r)fiktion. Literarische Verfahren der Selbstkonstruktion. Bielefeld: Aisthesis, 2012.

Woodmansee, Martha. The Author, Art, and The Market. Rereading the History of Aesthetics. New York: Columbia University Press, 1994. 